\title{
Ultrafast spectrum observation based on visualized spectro-temporal analyzer (ViSTA)
}

\author{
Chi Zhang, Xiaoming Wei, Jianbing Xu, P. C. Chui, and Kenneth K. Y. Wong* \\ The Photonic Systems Research Laboratory, Department of Electrical and Electronic Engineering, \\ The University of Hong Kong, Pokfulam Road, Hong Kong \\ *Email: kywong@eee.hku.hk
}

\begin{abstract}
We present an ultrafast optical spectrum analyzing modality known as visualized spectro-temporal analyzer, which leverages the time-lens focusing mechanism, can realize frame rate as high as $100 \mathrm{MHz}$, with $0.02-\mathrm{nm}$ resolution and $-30-\mathrm{dBm}$ detection sensitivity.

(C) 2013 Optical Society of America

OCIS codes: (110.6915) Time imaging; (190.4370) Nonlinear optics, fibers; (300.6530) Spectroscopy, ultrafast.
\end{abstract}

\section{Introduction}

Real-time optical spectrum analysis is an essential tool in observing ultrafast phenomena [1], such as the instantaneous linewidth of a swept-source [2,3], and the dynamic monitoring of a laser cavity. However, conventional optical spectrum analyzers (OSAs) are incapable of operating at high speed [4]; a more recent modality all-optically stretched the spectrum in time domain, but limited by the required input condition and the detection sensitivity [5]. According to the space-time duality, the time-lens focusing can achieve the transformation between time and wavelength [6]. For an arbitrary waveform within the time-lens effective range, it can relocate it according to its spectrum, and change the energy diverging process into converging, thus detection sensitivity would be greatly enhanced.

In this paper, we demonstrate this novel real-time spectrum resolving modality, called visualized spectro-temporal analyzer (ViSTA), with frame rate of $100 \mathrm{MHz}$. For the first time, we verify its applications in observing the dynamic spectrum of a Fourier domain mode-locked (FDML) laser.

\section{Experiment and Results}

Let us consider a scenario of an optical field with dynamic arbitrary spectrum along the time axis as shown in Fig. 1a. Based on conventional OSA measurement, the detailed evolution of all the spectral components will be intertwined. When this optical field is handled by the ViSTA system, which keeps sampling the spectrum in 10-ns intervals, these spectral components can be retrieved and lined up sequentially. Here, the frequency-to-time transformation of the ViSTA system is essentially a time-lens focusing process, according to the space-time duality [6] as shown in Fig. 1b, where the output dispersion is equal to the time-lens focal length. The parametric conversion can be easily achieved in a fiber-based $\chi^{(3)}$ system; the newly-generated idler will be imparted with twice the chirped range of the swept pump.
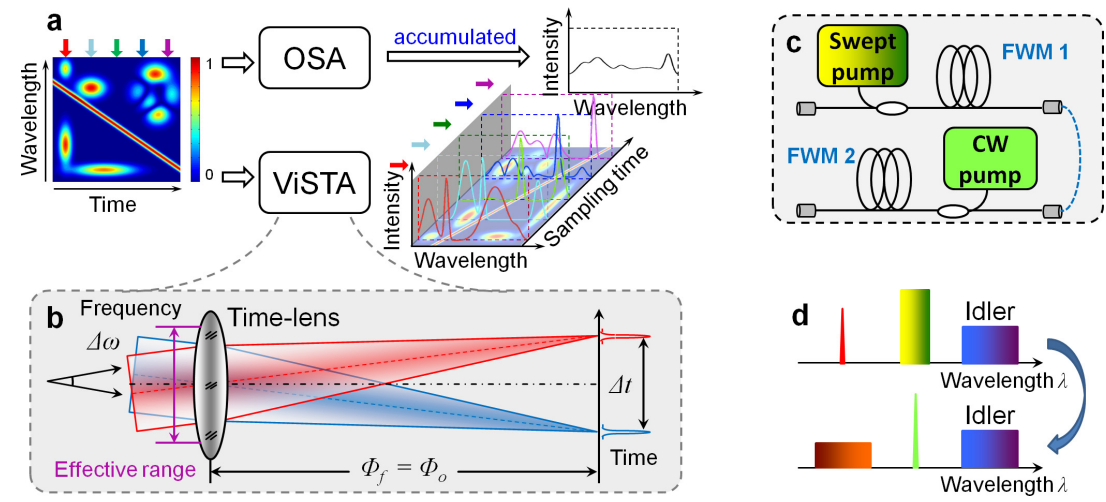

Fig. 1. ViSTA system: (a) basic operation of the ViSTA system, which captures its spectra at different time slots with 10-ns interval; (b) principle of ViSTA - the time-lens focusing. Different wavelengths are represented as parallel beams with different incident angles; (c) timelens is implemented by two-stage four-wave mixings (FWMs); (d) the spectral relation along the two stages of FWM. 
a

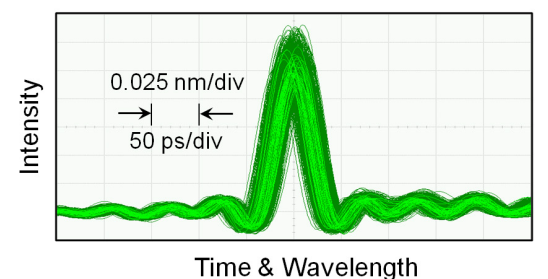

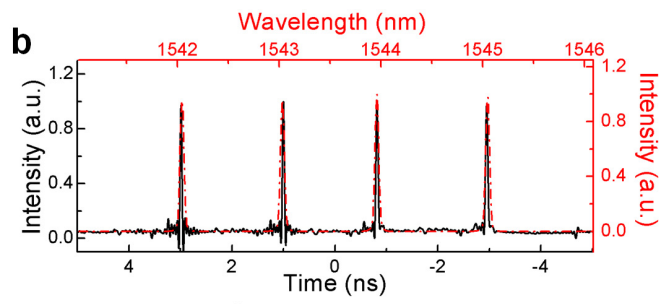
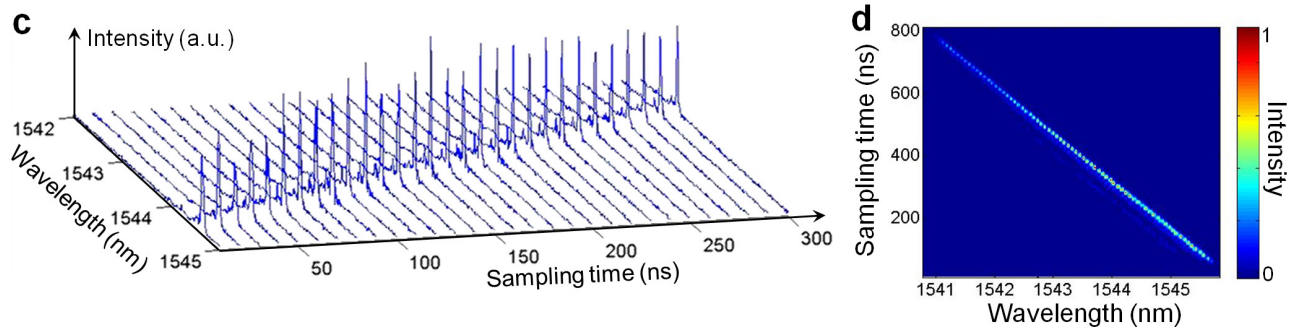

Fig. 2. Experimental results: (a) eye diagram of the ViSTA spectrum obtained from a single CW laser; (b) four CW sources were roughly separated by $1 \mathrm{~nm}$, dashed-dotted red line: spectra captured by a conventional OSA, and solid black line: spectra captured by ViSTA; (c) sequence of the FDML spectra movie frames acquired in a continuously sampling ViSTA system, with the frame rate of $100 \mathrm{MHz}$ (every 10 ns); (d) top view of the same FDML dynamic spectrum, and the color bar corresponds to the normalized spectral intensity.

The second stage of FWM performs as a phase conjugator (Fig. 1c and d), and the same spool of dispersive fiber is used in the time-lens and the output dispersion to achieve better dispersion compensation or pulse compression.

A single continuous-wave $(\mathrm{CW})$ source was first tested by ViSTA. Considering the higher-order dispersion and limited acquisition bandwidth $(16 \mathrm{GHz})$, the sharpest electrical pulse width was $40 \mathrm{ps}$, as the accumulated eye shape shows in Fig. 2a, which corresponds to the single-shot wavelength resolution of $0.02 \mathrm{~nm}$. The wavelength range of ViSTA was tested with four 1-nm spaced wavelengths, and the single-shot result is shown in Fig. 2b. As a comparison, the same signal was launched into the OSA with a resolution of $0.06 \mathrm{~nm}$, as shown in Fig. 2b. As a demonstration of the ultrafast real-time spectrum analyzing capability of the ViSTA system, we used it to capture the instantaneous spectrum of a fast swept-source, a FDML laser [2]. Here, the FDML laser was constructed with a long fiber ring cavity (5-km SMF), a semiconductor optical amplifier (SOA), and a fiber Fabry-Perot tunable bandpass filter. The swept range from $1541 \mathrm{~nm}$ to $1546 \mathrm{~nm}$ was filtered out, and evolution of the spectrum was obtained by the ViSTA (Fig. 2c). Figure $2 \mathrm{~d}$ shows the top view of the measured FDML spectra, with the average linewidth of $0.08 \mathrm{~nm}$.

\section{Conclusion}

In conclusion, we reported a novel ultrafast spectrum analyzing modality, ViSTA, achieving the frame rate of $100 \mathrm{MHz}$ based on the time-lens focusing technique. With focal dispersion of $2 \mathrm{~ns} / \mathrm{nm}$, this ViSTA system has demonstrated a spectral resolution of $0.02 \mathrm{~nm}$ within a 5-nm wavelength range. In order to verify this ultrafast performance, we have used the ViSTA to measure the dynamic spectrum of a FDML swept-source. We expect that ViSTA will find numerous applications in areas where rapid spectral data acquisition is important, especially of non-repetitive events.

\section{Acknowledgment}

The work described in this paper was partially supported by grant from the Research Grants Council of the Hong Kong Special Administrative Region, China (project HKU 717212E). The authors also acknowledge Sumitomo Electric Industries for providing the highly-nonlinear dispersion-shifted fiber (HNL-DSF).

\section{References}

1. D. Derickson, Fiber Optic, Test and Measurement (Prentice Hall, 1998).

2. R. Huber, M. Wojtkowski, and J. G. Fujimoto, "Fourier Domain Mode Locking (FDML): A new laser operating regime and applications for optical coherence tomography," Opt. Exp., 17, 5691-5697, 2009.

3. B. R. Biedermann, W. Wieser, C. M. Eigenwillig, T. Klein, and R. Huber, "Direct measurement of the instantaneous linewidth of rapidly wavelength-swept lasers," Opt. Lett., 35, 3733-3735, 2010.

4. A. B. Shafer, L. R. Megill, and L. A. Droppleman, “Optimization of the Czerny-Turner spectrometer," J. Opt. Soc. Am., 54, 879-887, 1964.

5. D. R. Solli, J. Chou, and B. Jalali, "Amplified wavelength-time transformation for real-time spectroscopy,” Nat. Photonics, 2, 48-51, 2008.

6. B. H. Kolner, "Space-Time Duality and the Theory of Temporal Imaging," IEEE J. Quantum Electron., 30, 1951-1963, 1994. 